Case

Report

\title{
Successful Repair of a Syphilitic Aortic Arch Aneurysm Accompanied by Serious Cerebral Infarction
}

\author{
Katsutoshi Sato, MD, PhD, Kiyoshi Chiba, MD, PhD, \\ Nobusato Koizumi, MD, PhD and Hitoshi Ogino, MD, PhD
}

\begin{abstract}
We present a 52-year-old male with a syphilitic aortic arch aneurysm accompanied by relevant extensive cerebral infarction. He was admitted to a local hospital for sudden loss of consciousness, where he was diagnosed with serious cerebral infarction. During his treatment, a multilocular aortic arch aneurysm involving the arch vessels was found incidentally. He was transferred to our hospital for surgical treatment. A preoperative routine laboratory test for syphilis was highly positive, which suggested that the aneurysm was likely caused by syphilis and the cerebral infarction was also induced by the involvement of syphilitic aortitis or arteritis. After 2 weeks of antibiotic therapy for syphilis, total arch replacement was performed successfully using meticulous brain protection with antegrade selective cerebral perfusion and deep hypothermia. He recovered without any further cerebral deficits. The pathological examination of the surgical specimen showed some characteristic changes of syphilitic aortitis.
\end{abstract}

Keywords: arch replacement, syphilitic aneurysm, cerebral infarction

\section{Introduction}

Total arch replacement with antegrade selective cerebral perfusion (SCP) and deep hypothermia for a syphilitic arch aneurysm was carried out successfully for a rare case of cerebral infarction due to occlusion of the left internal carotid artery (LICA) and severe stenosis of the left common carotid artery (LCCA), presumably caused by syphilitic aortitis or arteritis. We report on the clinical findings of syphilitic aortic arch aneurysm accom-

Department of Cardiovascular Surgery, Tokyo Medical University, Tokyo, Japan

Received: June 23, 2013; Accepted: October 10, 2013 Corresponding author: Katsutoshi Sato, MD, PhD. Department of Cardiovascular Surgery, Tokyo Medical University, 6-7-1 Nishishinjuku, Shinjuku-ku, Tokyo 160-0023, Japan

Email: satoktts-cvs@umin.ac.jp

(C)2014 The Editorial Committee of Annals of Thoracic and Cardiovascular Surgery. All rights reserved. panied by serious cerebral infarction and describe our procedure for the secure repair of the arch aneurysm.

\section{Case Report}

A 52-year-old man with an aortic arch aneurysm and sudden-onset extensive cerebral infarction was referred to our hospital for further examination and surgical treatment. He had suddenly developed loss of consciousness and had been urgently admitted to a local hospital, where he had been diagnosed with critical infarction of the left cerebral hemisphere on computed tomographic scanning (CT-scan) (Fig. 1). He had received intensive treatment for extensive cerebral infarction, and his condition had been stabilized with severe paralysis on the right side of his body and motor agraphia. An aortic arch aneurysm had been suspected based on chest X-ray. The CT-scan revealed a multilobular aneurysm on the aortic arch involving the arch vessels. The maximum diameter was $68 \mathrm{~mm}$ (Fig. 2). One month after the onset of his 


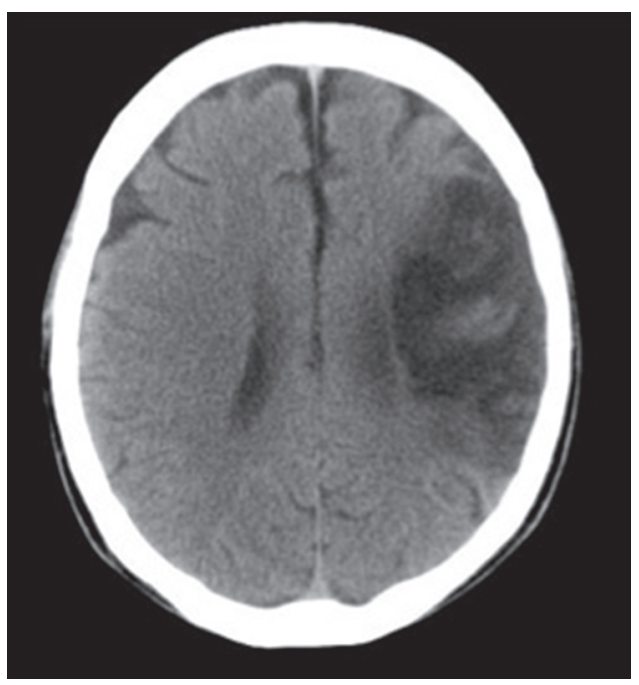

Fig. 1 Computed tomography of the brain showed a large cerebral infarction in the left middle cerebral artery territory.

symptoms, he was transferred to our hospital for surgical treatment of the arch aneurysm.

At admission, he had neither a previous history nor symptoms of syphilis; however, a routine laboratory test for syphilis before surgery was highly positive (rapid plasma regain test $>600 \mathrm{RU}$, treponema pallidum latex agglutination test $>30000 \mathrm{mU} / \mathrm{ml}$ ). In addition, the multilobular shape of the aneurysm was similar to a syphilitic aneurysm. Consequently, the arch aneurysm was considered to be due to syphilis. At first, antibiotic therapy with $2 \mathrm{~g} /$ day of ceftriaxone sodium was carried out for 2 weeks before surgery to treat the highly active syphilis.

A preoperative CT-scan demonstrated complete occlusion of the LICA and severe stenosis of the LCCA orifice (Fig. 2B). Cerebral perfusion scintigraphy showed critically low perfusion of the left cerebral hemisphere. Diamox brain perfusion scintigraphy revealed decreased function of the right cerebral hemisphere as well.

Sixtynine days after the onset of cerebral infarction, total arch replacement was carried out through a median sternotomy. Cardiopulmonary bypass was estaqwblished with our routine double arterial cannulation via the right axillary artery (RAxA) and the ascending aorta. Venous drainage was performed through the right atrium. Deep hypothermia at $20^{\circ} \mathrm{C}$ was employed because of the severe cerebral infarction. The three arch vessels were clamped and circulatory arrest was induced. The mid-ascending aorta was clamped and cold blood cardioplegia was infused antegradely. The arch aneurysm was opened. The aneurysmal wall was moderately thickened with some inflam-

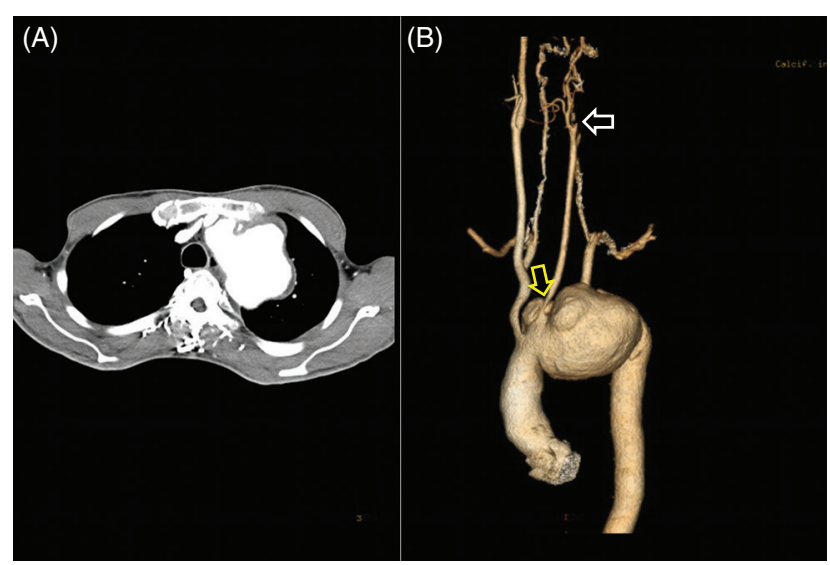

Fig. 2 (A) Computed tomography revealed a multilobular aneurysm on the aortic arch. (B) The aortic arch had aneurysmal changes from the distal part to the brachiocephalic artery, whose orifice was compressed by the aneurysm. The LCCA and LSCA were involved near the aneurysm, and the orifice of LCCA was severely stenosed (bottom arrow). The LICA was totally occluded toward the middle cerebral artery (top arrow). LCCA: left common carotid artery; LICA: left internal carotid artery; LSCA: left subclavian artery.

mation presumably due to syphilitic aortitis. The aneurysmal wall was resected as much as possible. The orifice of the LCCA was severely stenosed due to syphilitic aortitis and arteritis (Fig. 3). The short-segment was then resected and the cannula was inserted for selective cerebral perfusion (SCP), which was established completely with additional perfusion of the left subclavian artery (LSCA). The SCP flow was $12-15 \mathrm{ml} / \mathrm{kg} / \mathrm{min}$, which was relatively higher than the standard flow of $10-12 \mathrm{ml} / \mathrm{kg} / \mathrm{min}$ and was used to maintain higher cerebral perfusion pressure for more meticulous brain protection. The descending aorta was divided at the distal end of the aneurysm through the aneurysm. The distal anastomosis was made with a continuous 4-0 polypropylene suture with outside felt-strip reinforcement. The prosthesis was a 22-mm multibranched woven Dacron graft. The duration of circulatory arrest of the lower half of the body was 48 minutes. The three arch vessels were reconstructed with continuous 5-0 polypropylene sutures. Rewarming was initiated. Finally, the proximal aortic anastomosis was created with a continuous 4-0 polypropylene suture with outside felt-strip reinforcement. The duration of SCP, extracorporeal circulation and operation were 104, 202 and 431 minutes, respectively. The pathology of the aneurysmal wall revealed diffuse infiltration of lymphocytes and plasma cells, which is 

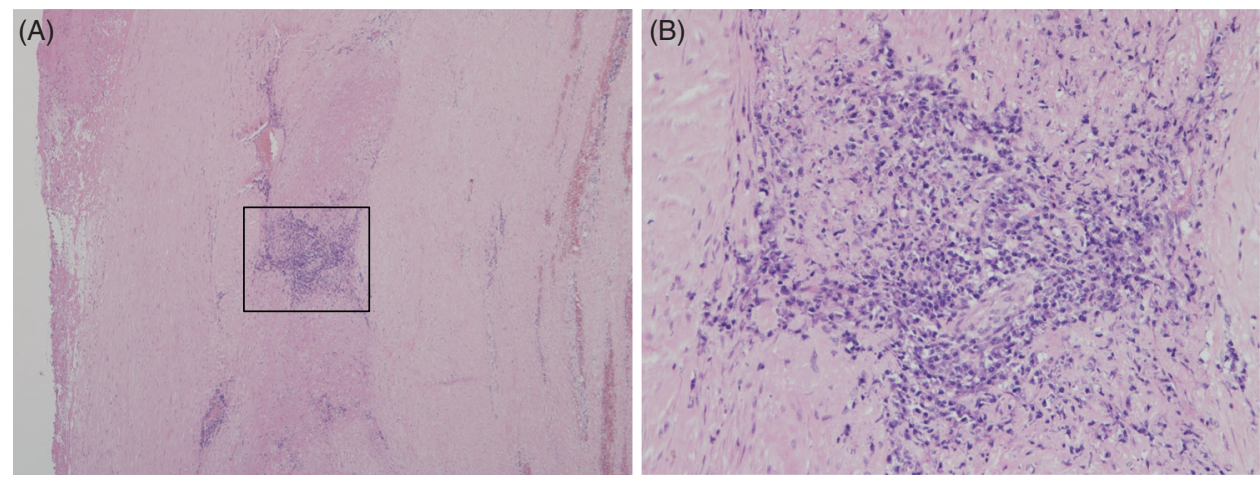

Fig. 3 The pathology of the aneurysmal wall around the origin of the left common carotid artery, revealed diffuse infiltration of lymphocytes and plasma cells (hematoxylin and eosin stain; A: $\times 4, \mathbf{B}: \times 20)$.

characteristic of syphilitic aortitis. No bacteria, fungi or spirochetes could be detected in the aneurysmal wall (Fig. 3).

He recovered without any new neurologic deficits or any symptoms of syphilis, and was transferred to an outside hospital for further rehabilitation on postoperative day 16 . We requested a serological test for syphilis and the additional administration of antibiotic therapy if necessary.

\section{Discussion}

Although syphilis is one of the etiologies of aortic aneurysm, the incidence is very low. Recently, the incidence has decreased remarkably, particularly in developed countries such as Japan. Cardiovascular syphilis is a manifestation of tertiary syphilis. ${ }^{1,2)}$ Our patient did not have a previous history or physical findings of syphilis; however, both the RPR test and TPLA test were highly positive before surgery. The aneurysm also showed a multilobular shape. The pathology of the surgical spacemen revealed diffuse infiltration of lymphocytes and plasma cells in the aortic wall. The aneurysm was consequently thought to be due to syphilis. Because of the highly positive treponemal serologic test, his syphilis appeared to be active. Ceftriaxone sodium was administrated for 2 weeks before surgery, because of his penicillin allergy. ${ }^{3)}$ Despite the fact that a person's titer as detected by the serum syphilis test does not change for several months, ${ }^{3)}$ we nevertheless performed surgery as soon as possible given the size and shape of the aneurysm.

In general, syphilitic aortitis is frequently associated with tertiary syphilis and often occurs in the ascending aorta, which results in aortic insufficiency, coronary ostial stenosis and ascending aortic aneurysm. ${ }^{1,2)}$ In this case, the ascending aorta was not dilated, and the aortic valves and the coronary arteries including the coronary artery ostia were not involved. The distal arch was dilated and involved the arch-vessels resulting in a severe cerebral infarction. There have been few reports on a syphilitic aortic aneurysm resulting in stroke. ${ }^{4-6)}$ Furthermore, total arch replacement with SCP and deep hypothermia for such cases has never been reported.

The patient had not developed neurological symptoms until the cerebral infarction. The orifice of the LCCA was severely stenosed due to the involvement of syphilitic aortitis. The LICA was totally occluded. Arterial dissection may occur at any intracranial site, resulting in cerebral infarction. Syphilis is thought to be one etiology of arterial dissection.6,7) Our case did not have any arrhythmia, ischemic heart disease or valvular heart disease that could serve as a source of cerebral emboli. It was undeniable that thrombus embolism from the aorta caused the LICA occlusion; however, dissection of the intracranial artery due to syphilis might be attributed in part to the LICA occlusion.

In terms of brain protection during arch repair, the preoperative CT-scan showed that cerebral blood flow was restricted due to complete occlusion of the LICA. Furthermore, the LCCA and LSCA were involved near the aneurysm, and the orifice of LCCA was severely stenosed. The aortic arch had aneurysmal changes from the distal part of the brachiocephalic artery, whose orifice was also compressed by the aneurysm. Cerebral perfusion scintigraphy showed low perfusion of the bilateral cerebral hemispheres. The patient was considered to be 
at extremely high risk of additional cerebral deficits during total arch replacement.

Bachet $^{8)}$ and Kazui ${ }^{9)}$ demonstrated the superiority of SCP for cerebral protection during aortic arch repair. However, Kazui reported that an old cerebral infarction is a significant risk factor for neurological dysfunction, even with SCP and deep hypothermia. ${ }^{9)}$ However, deep hypothermia is still a gold-standard technique for vital organ protection including the brain. ${ }^{10)}$ Simple deep or profound circulatory arrest is still used widely as a reliable technique for aortic arch surgery. Therefore, SCP with deep hypothermia was used in our case. During arch repair, we meticulously regulated the SCP flow to maintain an arterial pressure above $50 \mathrm{mmHg}$, and this required an approximately $20 \%$ increase in SCP flow. The level of hemoglobin was also maintained around $10 \mathrm{~g} / \mathrm{dl} .{ }^{11)}$

Another useful technique for brain protection is RAxA perfusion. ${ }^{12)}$ In this case, the perfusion of the right hemisphere was important, because the LICA were completely occluded. We have routinely used RAxA perfusion for aortic arch repair. With this technique, all of the surgical procedures are performed smoothly with reliable cerebral protection and no cerebral hypoperfusion or embolism. We believe that RAxA perfusion might have been particularly useful in this case, since the arch aneurysm compressed the innominate artery resulting in low perfusion of the right cerebral hemisphere.

Regional tissue oxygen saturation $\left(\mathrm{rSO}_{2}\right)$ was continuously monitored by the in vivo optical spectroscopy (INVOS) system. ${ }^{13}$ ) The level of $\mathrm{rSO}_{2}$ was maintained during SCP. As a result, the patient recovered smoothly without any new cerebral deficits.

\section{Conclusions}

We experienced a rare case of syphilitic aortic arch aneurysm accompanied by relevant extensive cerebral infarction. Total arch replacement was successfully performed with meticulous brain protection that required relatively higher SCP flow using RAxA cannulation and deep hypothermia.

\section{Disclosure Statement}

We have no conflict of interest to disclose with respect to this presentation.

\section{References}

1) Heggtveit HA. Syphilitic aortitis. A clinicopathologic autopsy study of 100 cases, 1950 to 1960 . Circulation 1964; 29: 346-55.

2) Roberts WC, Ko JM, Vowels TJ. Natural history of syphilitic aortitis. Am J Cardiol 2009; 104: 1578-87.

3) Spornraft-Ragaller P, Abraham S, Lueck C, et al. Response of HIV-infected patients with syphilis to therapy with penicillin or intravenous ceftriaxone. Eur J Med Res 2011; 16: 47-51.

4) Park MG, Oh SJ, Park KP. Multiple cerebral infarctions associated with syphilitic aortitis. Korean J Stroke 2011; 13: 31-3.

5) Umesue M, Durairaj M, Matalanis G, et al. Surgical repair of a syphilitic aneurysm of the distal arch and descending aorta. Asian Cardiovasc Thorac Ann 2006; 14: $150-2$.

6) Nakane H, Okada Y, Ibayashi S, et al. Brain infarction caused by syphilitic aortic aneurysm. A case report. Angiology 1996; 47: 911-7.

7) Iida H, Kusumi M, Sagiuchi T, et al. Cerebral infarction and fluid collection due to an enlarged dissecting aneurysm of the middle cerebral artery-case report. Neurol Med Chir (Tokyo) 2004; 44: 535-9.

8) Bachet J, Guilmet D, Goudot B, et al. Antegrade cerebral perfusion with cold blood: a 13-year experience. Ann Thorac Surg 1999; 67: 1874-8; discussion 1891-4.

9) Kazui T, Washiyama N, Muhammad BA, et al. Total arch replacement using aortic arch branched grafts with the aid of antegrade selective cerebral perfusion. Ann Thorac Surg 2000; 70: 3-8; discussion 8-9.

10) Griepp RB, Stinson EB, Hollingsworth JF, et al. Prosthetic replacement of the aortic arch. J Thorac Cardiovasc Surg 1975; 70: 1051-63.

11) Ogino H, Sasaki H, Minatoya K, et al. Evolving arch surgery using integrated antegrade selective cerebral perfusion: impact of axillary artery perfusion. J Thorac Cardiovasc Surg 2008; 136: 641-8; discussion 948-9.

12) Numata $S$, Ogino $H$, Sasaki $H$, et al. Total arch replacement using antegrade selective cerebral perfusion with right axillary artery perfusion. Euro J Cardiothorac Surg 2003; 23: 771-5.

13) Kochi K, Sueda T. Inflammatory aortic arch aneurysm with total occlusion of cervical branches. Ann Thorac Cardiovasc Surg 2004; 10: 51-3. 